\title{
Los intereses de los países ricos y el desarrollo del Tercer Mundo: La República Federal de Alemania
}

\section{LA POSIGIÓN GENERAL DE LA REPÚBLICA FEDERAL DE Alemania frente al Tercer Mundo*}

La República Federal de Alemania es un fruto del orden internacional que surgió después de la Segunda Guerra Mundial. El intento de dominación mundial por parte de Hitler había dejado a Europa dividida y en ruinas y el imperio germano destruido; la República Federal de Alemania fue fundada en el lado occidental de la cortina de hierro.

El conflicto este-oeste dominó la política exterior de Alemania Occidental, hasta que la détente y la Ostpolitik de Willy Brand abrieron las puertas de las Naciones Unidas a ambos estados alemanes en 1973, lo que los convirtió en miembros más o menos normales del sistema global.

La posición general de la República Federal de Alemania frente al Tercer Mundo está todavía fuertemente determinada por los intereses políticos y estratégicos de occidente. Sin embargo, sus intereses económicos con respecto al aprovisionamiento y a los mercados abarcan una gama de alcance mucho más global, y comprenden tanto los países occidentales como los países socialistas y el Tercer Mundo. En un nivel diferente, el impulso humanitario para contribuir al desarrollo en términos de las necesidades básicas, los derechos humanos y la autode-

*Este trabajo fue escrito para un proyecto conjunto de investigación del Institut of Development Studies (Universidad de Sussex, Brigton) el Overseas Development Institut (Londres) y el Overseas Development Council (Washington), sobre "Los intereses de los países ricos y el desarrollo del Tercer Mundo. Este proyecto pretende contribuir a la discusión realizada en diversas partes del mundo por la COMISIÓN INDEPENDIENTE SOBRE. TEMAS DE DESARROLLO INTERNAGIONAL, encabezada por Willy Brandt y los preparativos de la Tercera DÉCADA DEL Desarrollo de la ONU, si bien no existen conexiones ni responsabilidades formales.

Los trabajos sobrc otros países: Los Estados Unidos, el Reino Unido, Francia, Holanda, los Países Escandinavos, Japón, Australia,'Canadá y los países socialistas de Europa del Este. Además de aquel sobre Alemania Occidental serán publicados próximamente junto con un enfoque más amplio sobre los intereses mutuos entre el Norte y el Sur.

El trabajo aquí publicado podrá ser objeto de pequeñas revisiones en su versión final en inglés. 
pendencia otorgan un colorido especial a su política con respecto a los países en desarrollo.

Debido al hecho de que Alemania ya había perdido sus colonias después de la primera guerra mundial, los lazos ex coloniales son mehos importantes para Alemania Occidental que lo que lo son para sus socios de Europa Occidental.

La falta de una experiencia reciente de descolonización y, si comparamos con los EE.UU., de una guerra de Vietnam, explican en parte la causa de que la corriente principal de pensamiento acerca del desarrollo y del subdesarrollo económicos de los países del Tercer Mundo sea bastante conservadora. No sin razón, Alemania Occidental es considerado como un desinhibido exponente de la línea dura frente a los temas económicos Norte-Sur. El gobierno, los partidos que lo respaldan y la oposición parlamentaria, como también la inmensa mayoría de los medios de comunicación y de los economistas perciben que los intereses alemanes son mejor servidos por el orden económico internacional existente. Se admite, por supuesto, la necesidad de mejorarlo, pero no se vislumbra esencialmente un "nuevo" orden por el que valga la pena luchar.

El credo liberal afirma que el intercambio económico siempre satisface los intereses de ambas partes. Al analizar la interacción económica Norte-Sur es más frecuente suponer que demostrar este interés mutuo. Las demandas de los voceros del Tercer Mundo que se oponen a esta filosofía básica son interpretados a menudo como mal aconsejadas, prejuiciadas ideológicamente y como si no estuviesen en consonancia con los intereses de los países en desarrollo.

Existe la creencia básica de que la fuerza económica de Occidente es la que saca de la miseria y del subdesarrollo al Tercer Mundo, y que se podría y habría que hacer más para utilizar esta fuerza. Sin embargo, cualquiera crítica radical que relacione el subdesarrollo con la estructura del orden económico internacional es vista con incomprensión, cuando no con abierta hostilidad.

La posición conservadora açtual frente al Tercer Mundo es desafiada a veces por radicales, ecologistas y escépticos, pero no hasta el mismo grado que en otros países europeos. El orden existente ha servido tan bien a Alemania Occidental, que no se observa en este país ninguna inclinación en favor de "otro desarrollo".

Es también muy difícil comprender la idea de que más control estatal y más autoconfianza nacional, como asimismo más relaciones económicas intergubernamentales, deberían proporcionar las soluciones para salir del subdesarrollo. Para los alemanes, a causa de su experiencia en el Tercer Reich, el nacionalismo y un poderoso control 
gubernamental representan preferentemente una incitación a la guerra y a la represión totalitaria, y para ellos, los tratados económicos intergubernamentales son las causas de los torpes y fallidos intentos para manejar la crisis en los años treinta. Tiende a afirmar estos puntos de vista la poco exitosa reconstrucción económica de la República Democrática Alemana en un orden internacional de tipo económico, que ha ido de la mano con la supresión de las libertades civiles. Si los países en desarrollo demandan ahora más control gubernamental por causa del desarrollo económico y de la emancipación nacionalista, ello es visto como un paso atrás en vez de adelante por aquellos que han vivido los últimos cincuenta años de la historia alemana y que tienden a juzgar el mundo por sus patrones.

Con respecto a esto se puede subrayar un punto importante: existe un fuerte interés alemán por la mantención y el aumento de los elementos globalistas en la estructura general del mundo con respecto al campo económico, bajo la bandera del libre comercio, que ha sido siempre el estandarte de los fuertes. En tanto el Tercer Mundo también mantenga un interés en estos elementos: acceso a los mercados, inversión en la producción de materias primas, investigación sobre las fuentes alternativas de energía, transferencia de tecnología, ayuda y préstamos externos, se puede esperar razonablemente que exista cierta mutualidad de intereses. Por encima de todo, deben citarse aquí los objetivos más generales, tales como la mantención de la paz, la eliminación de la carrera armamentista y la restauración o protección del equilibrio ecológico mundial.

A fin de conseguir sus intereses globales, Alemania tiene abiertas dos sendas ante sí, que describe muy bien Stanley Hoffmann en su alternativa propuesta para los EE.UU.: Primacy or World Order. La estrategia de una "primacía compartida" junto con los demás países occidentales, implicaría un aumento de la convocatoria trilateral, una línea defensiva en contra de los desposeídos y del bloque oriental, una cierta flexibilidad en la cooptación de países individuales "importantes" del Tercer Mundo y la defensa del orden económico internacional establecido. Las consideraciones de una política pragmática (Realpolitik) parecen apuntar en esa dirección, principalmente por causa de la "escalada piramidal" bastante exitosa que hubo en la República Federal después de la segunda guerra mundial, a la que ya nos referimos. Por otra parte, la estrategia del "orden mundial" parece mucho más gravosa, ya que implicaría una cooperación intergubernamental formal y más dirigismo en materias económicas. Sin embargo, a pesar del clamor de los partidarios de la línea dura, también en la República Federal de Alemania se escucha la súplica de Stanley Hoffmann con respecto de 
los EE.UU., en favor de esta estrategia. En un discurso programático, el Ministro de Relaciones Exteriores declaró recientemente que es necesaria una "revolución en las doctrinas de política exterior": "tenemos que construir un orden de cooperación que sea considerado justo por ambos lados, también por el lado de los países en desarrollo" ${ }^{\prime \prime}$ enfatizando, sin embargo, que la reforma del sistema económico debe conformarse a las reglas económicas del mercado.

La meta de construir un verdadero orden mundial se ve amenazada en épocas de tensiones entre el Este y el Oeste. La identidad de bloque en ambos lados de la frontera alemana-alemana es probablemente más fuerte que en los países que tienen una identidad nacional intacta. Por consiguiente, es comprensible que los intereses alemanes sean definidos a menudo en términos de intereses de bloque, los que por su naturaleza se inclinan a un juego del tipo "suma cero", y que se tienda a invalidar otros menos necesarios y con objetivos más difusos a largo plazo.

Existen incluso voces que piden una fuerza militar conjunta de Occidente $^{2}$, y el Ministro de Cooperación Económica, Rainer Offergeld, ha tenido que declarar en el Parlamento que "la política de desarrollo no es una política de alianzas a través de medios diferentes"3.

Además de los niveles en que operan el bloque globalista y el bloque occidental, existen a nivel de la Comunidad Europea un fuerte conjunto de intereses que hay que tomar en cuenta al explorar las esferas de la mutualidad y del conflicto en las relaciones Norte-Sur.

Es en el nivel europeo donde se puede obtener un entendimiento frente a la demanda para controlar las corporaciones transnacionales por parte de los países del Tercer Mundo, para aumentar su posición negociadora y para controlar efectivamente su propia economía. De acuerdo al informe Tindeman de 1975 sobre el futuro de la Comunidad Europea, uno de los motivos más fuertes para la integración europea es el creciente sentido de una "doble espiral de impotencia": hacia el exterior porque ya no existe la edad eurocéntrica, y hacia el interior porque los procesos económicos ya no pueden seguir siendo controlados por los gobiernos nacionales.

Esto suena muy semejante a las afirmaciones del Tercer Mundo. De allí que las actitudes de Alemania Occidental y del Tercer Mundo tendientes a la autodependencia (colectiva), al control estatal, a la

\footnotetext{
${ }^{1}$ Genscher (1979).

${ }^{2}$ Todenhöfer (1979).

${ }^{3}$ Vorwärts, 31.I. 1980, 14.
} 
prioridad de la integración interna y a una cierta distancia del gran poder bipolar resulten no estar tan distanciadas entre sí, si es que se considera el nivel europeo, como parecen sugerirlo los niveles de bloque globales y occidentales.

La lista de los intereses alemanes generales que debe ser considerada en el contexto Norte-Sur estaría incompleta si se confinara a los intereses globales, a los intereses de bloque y a los intereses de cómo ganar o recuperar el control del propio destino, tanto en el nivel europeo como en el nacional. Debe añadirse un cuarto campo de interés en el nivel subnacional, que es la preocupación directa con la pobreza, la marginalidad y los derechos humanos.

Esta preocupación humanitaria no conduce por sí misma a una fácil articulación, pero a pesar de su carácter motivacional bastante difuso e indirecto, no puede ser ignorada como la fuerza conductora que está detrás de las antesalas para obtener ayuda, ni como un desafío para mostrar o restaurar la decencia moral, ya que no la superioridad del propio sistema, ni tampoco como la percepción de una amenaza hacia la propia comunidd (frente al desempleo, a los ghettos de trabajadores extranjeros en las ciudades alemanas, y a los nuevos miembros menos prósperos de la Comunidad. Europea). Existe el temor fundamental de que el progreso reciente con respecto a los derechos humanos, la igualdad y la democracia como principios universales pueda ser desacreditado nuevamente. Después de todo, el pasado alemán nazi yace tan solo 35 años atrás y la vuelta de las ideologías antiigualitarias o la emergencia de nuevas ideologías (por ejemplo, la tecnocracia o la sociobiología) no es imposible, si persisten estructuras socioeconómicas tan desiguales.

Este balance general de los campos de interés para determinar la posición de la República Federal de Alemania frente al desarrollo del Tercer Mundo muestra un patrón que no es raro en el análisis de las políticas cuando se diferencian los "intereses nacionales": cada campo de intereses - los globalistas tanto como los de bloque, los intereses de control en los niveles nacionales y europeos y los intereses humanitarios- tiene su propia legitimidad y además, una cierta base social y una justificación ideológica propia. Su búsqueda, sin embargo, puede ser ciega hasta el punto de ser homicida, y si se lleva demasiado lejos, pierde de vista a los demás. La tarea permanente de todo gobierno es balancear estos intereses. No se puede alcanzar ninguna combinación "óptima", que lo sea para todos, porque no hay implicados juicios de valor individuales y de grupo, que no pueden ser agregados a una función de bienestar social consistente. Sin embargo, puede presumir- 
se en forma segura un cierto mínimo, bajo el cual sería difícil alcanzar un consenso democrático.

\section{II, OPINIÓN PÚBLICA Y FORMULACIÓN DE POLÍIICAS}

La República Federal de Alemania experimenta una mezcla de sentimientos frente al Tercer Mundo. Se considera justo y adecuado hacer algo contra el hambre y la pobreza en el mundo y, como ya se dijo antes, este esfuerzo para comportarse en forma decente puede ser interpretado como un interés difuso en un genuino desarrollo. Por otra parte, mientras más intolerables parezcan las condiciones de vida en el Tercer Mundo, más peligro existe de que aquellos países caigan en un caos que amenace las islas de prosperidad ${ }^{4}$. Sin embargo, el miedo y la ansiedad llevan al deseo de dominar la amenaza y de postergar, en vez de amparar, la emancipación y el desarrollo del Tercer Mundo ${ }^{5}$. Cuando se trata de influir en las políticas, se juegan los intereses más compactos y francos en esta ambigüedad existente en la opinión pública entre solidarizar con el Tercer Mundo o dominarlo.

Estudios recientes de la opinión pública indican un creciente interés en la política de desarrollo y una actitud general cada vez más positiva hacia la ayuda ${ }^{6}$. Se considera que la importancia de la ayuda para el desarrollo está destinada a aumentar en el futuro ${ }^{7}$. Una parte de la población adulta (44\%) declara estar lista para sacrificar un cierto porcentaje de aumento en sus ingresos en favor de la ayuda. Al mismo tiempo, sin embargo, la brecha entre las actitudes positivas hacia la ayuda en general y hacia las políticas de desarrollo alemanas se está ensanchando; especialmente entre la generación joven y aquellos que poseen educación secundaria y superior. La gran mayoría de este último grupo está a favor de la ayuda en general (93\%), pero sólo una pequeña mayoría (57\%) demuestra una opinión positiva frente a las políticas de desarrollo alemanas, habiéndose ensanchado la brecha a $36 \%$, de $21 \%$ en $1975^{8}$. Esto refleja el espíritu altamente crítico de la comunidad del desarrollo, que la componen aquellos que están interesados activamente en los problemas del desarrollo, los grupos de acción y las voces académicas no conformistas, que argumentan que más cantidad de lo mismo no es solución. A ellos les parece que un mayor número de exportaciones de una tecnología a menudo inadecuada,

${ }^{4}$ Ver Hager y Noelle-Neumann, 93-94.

${ }^{5}$ Duve 1971 .

${ }^{6}$ Resultados muy similares por INFRATEST y EMNID; ver Ministerio de Cooperación Económica (1979 c) y EMNit1).

${ }^{7}$ EMNID, 11.

${ }^{8}$ Ministerio de Cooperación Económica $(1979$ c), 5. 
más inversión en la producción de automóviles particulares, más ayuda de burocracia a burocracia, más importaciones de manufacturas de mano de obra barata y también de productos como frutas, verduras, alimentos para perros y flores desde los países en desarrollo semejan una estrategia para la perpetuación, si no para la universalización de la pobreza, combinada con pequeñas islas de prosperidad y estilos de vida cosmopolitas. Las políticas actuales hacia el Tercer Mundo no se ven muy afectadas por estos argumentos. Ellas están, en realidad, menos determinadas por grupos orientados hacia el desarrollo, que tienen intereses específicos y que presionan para que se realicen ciertas actividades respaldadas por una opinión pública comprometida con el desarrollo, que por el surgimiento de políticas económicas o exteriores generaless, cuya forma está moldeada por la constelación general de fuerzas dentro de la sociedad alemana y del sistema internacional.

El grupo económico más influyente en Alemania Occidental lo constituye el sector industrial exportador ${ }^{9}$, que ha tenido éxito en crear una "mística" general de exportaciones. A menudo se afirma que la competencia internacional es el objetivo final de toda política. El exitoso dinamismo del crecimiento orientado hacia las exportaciones en el período posterior a la segunda guerra mundial ha dado origen a la creencia de que el libre comercio y el libre movimiento de los factores contemplan el interés general tanto en Alemania como en el extranjero, y que el interés alemán primordial en el desarrollo mundial radica en el crecimiento de los mercados de exportación.

La presión en favor de los intereses económicos globalistas apenas logra ser mitigada dentro de la Federación de la Industria Alemana (Bundesverband der Deutschen Industrie), que agrupa las asociaciones nacionales de la rama industrial, a causa de que la presión proteccionista se ejerce primariamente en el nivel de la Comunidd Europea, que es donde se realizan las decisiones de políticas comerciales.

En el nivel nacional, la industria es secundada en sus antesalas de negocios orientadas hacia el libre comercio, el antidirigismo, y en favor de lo privado por la Federación de Comercio Mayorista Libre (Bundesverband des Deutschen Gross und Aussenhandels), que agrupa, entre otras, las firmas más importantes (la exportación la realizan en su mayoría las grandes firmas industriales en forma directa, sin usar intermediarios), la Federación de Bancos Alemanes (Bundesverband Deutscher Banken) y la Asociación de Industria y Comercio (Deutscher Industrie und Handelstag), una organización de muy alto nivel que agrupa las cámaras de comercio e industria locales y de los distritos.

\footnotetext{
${ }^{9}$ Para mucho de lo que sigue ver Kreile.
} 
En el lado proteccionista se puede encontrar a la Asociación Alemana de Agricultores (Deutscher Bauerverband) y a los grupos y entidades privadas y públicas que están afectados por las reestructuracionés industriales o agrarias en regiones agobiadas por problemas. La posición de los sindicatos y de su federación máxima (Deutscher Gewerkschaftsbund) es bastante compleja en lo que se refiere a las políticas para el desarrollo del Tercer Mundo. Durante largo tiempo, prevaleció el modelo de la gran coalición entre el capital, el trabajo y la dirección de la empresa dentro del ámbito de cada rama industrial. Durante la época del pleno empleo, la emigración fue bienvenida, incluso la de los países no pertenecientes a la Comunidad Europea (por razones que se explicarán más adelante en el capítulo sobre la emigración). En años recientes, con una persistente tasa de desempleo de alrededor del 3-4 por ciento, que es alta para los patrones alemanes, la posición de Alemania, que por lo general estaba en favor del libre comercio, de la inversión extranjera y de la emigración ha cambiado, para llegar a estar más de acuerdo con las ramas industriales.

Cada vez les parece más claro, no sólo a los trabajadores textiles sino también a ciertas subramas de los sindicatos metalúrgicos, que una reestructuración de la geografía industrial del mundo de acuerdo con las ventajas comparativas de una fuerza de trabajo barata y abundante disponible en el Tercer Mundo podría perjudicar en vez de ayudar a ambas partes: en los países industriales no perderían puestos de trabajo y en los países en desarrollo se congelarían los salarios a niveles muy bajos, a causa de la intensa competencia entre estos países por atraer inversiones privadas para actividades industriales de exportación. Consideraciones de este tipo han sido articuladas en discursos y declaraciones ${ }^{10}$, pero aún no han sido transformadas en prescripciones de políticas ni en cabildeos en una dirección específica.

Por el lado del gobierno, los diversos ministerios y agencias persiguen sus políticas en forma bastante independientes. La retórica y la línea general de argumentos en la política de la economía externa está dominada por el Ministerio Federal de Economía (Bundesministerium für Wirtschaft), un baluarte de la.filosofía neoliberal desde los primeros tiempos de la República Federal de Alemania, cuando Ludwig Erhard fue el primer ministro de economía. Es también responsable por las políticas sobre productos y energía, como también por las políticas antitrust.

\footnotetext{
${ }^{10}$ Ver Buschmann, Ministerio de Cooperación Económica (1978), Ih.ETU.
} 
El Ministerio de Relaciones Exteriores (Auswärtiges Amt) generalmente encabeza las delegaciones en las conferencias internacionales y extrae a la fuerza las fórmulas de compromiso; el Ministerio de Finanzas (Bundesministerium für Finanzen) junto con el Banco Central (Deutsche Bundesbank), maneja los asuntos monetarios internacionales, el Ministerio de Cooperación (Bundesministerium für wirtschaftliche Zusammenarbeit) maneja la asistencia técnica y financiera a los países en desarrollo y es responsable por gran parte de la cooperación científica y tecnológica, que en principio cae en el dominio del Ministerio de Investigación y Tecnología (B'undesministerium für Forschung und Technologie). El Ministerio de.Alimentación y Agricultura (Bundesministerium für Ernährung und Landwirtschaft) es responsable por las políticas agrarias y participa en su formulación en el nivel europeo; su programa de investigación agrícola es interesante para los países en desarrollo,

La coordinación se efectúa en comités interministeriales. Los funcionarios más antiguos hacen uso de los consejos de aquellos estudiosos interesados en el tema; ellos conocen a sus interlocutores en el Tercer Mundo $y$ han desarrollado formas rutinarias para armonizar posiciones sin interferir realmente con el dominio del otro. Sólo los temas más controvertidos alcanzan a llegar a los Ministerios, al Canciller y al Gabinete. La oficina del Canciller (Bundeskänzleramit) no muestra mayor interés en los temas del desarrollo.

Puesto que hay poca legisiación referente a la política económica internacional, el Parlamente (Bundestag) no es muy importante, excepto por una propensión general hacia la articulación de intereses y la formulación de políticas: los demócrata-cristianos se oponen a la coalición gubernamental de liberales y social-demócratas en la línea general de "libertad" versus "socialismo" (su slogan en la campaña para la elección general de 1976). Cualquier concesión a las demandas del Tercer Mundo, a las cuales pudiera ser atribuido el término "dirigismo", está por lo tanto abierta a ataque parlamentario. Además, las recientes actitudes soviéticas han conducido a los demócrata-cristianos a percibir el conflicto Norte-Sur como cada vez más dominado por el conflicto entre Este y Oeste ${ }^{11}$, un punto de vista que es compartido en parte por el gobierno y el público. Con una oposición conservadora en sus talones, el ya conservador gobierno, apoyado por la corriente principal de economistas profesionales, es virtualmente inmune contra el criticismo voceado por los que proponen un estilo de vida alternativo radical y por las posiciones estructuralistas, que insisten en que el

\footnotetext{
${ }^{11}$ Unión Demócrata Cristiana, 1 c.
} 
desarrollo del Tercer Mundo no es lo mismo que la "occidentalización".

En el proceso formal de la formulación de políticas, los únicos canales para la continua articulación de posiciones alternativas son unos pocos miembros del ala izquierda del Parlamento y algunos funcionarios, especialmente del Ministerio de Cooperación Económica, cuyo trabajo consiste en negociar con socios en el Tercer Mundo y con la comunidad de desarrollo al interior de Alemania.

Muchos de estos grupos están unidos a las iglesias alemanas (católicas y protestantes), cuyo rol en la formulación de políticas hacia el Tercer Mundo es importante. La Conferencia de las Iglesias sobre el Desarrollo en la República Federal de Alemania (Gemeinsame Kommision der Kirchen für Entwicklungsländerfragen) dirige regularmente informes al gobierno antes de las conferencias de UNCTAD y en similares ocasiones, y llama a dar respuestas más orientadas hacia el desarrollo y hacia las demandas de los países en desarrollo y a otorgar más seriedad a las preguntas efectuadas por el movimiento de estilos de vida alternativos.

Más aún, las iglesias alemanas protestantes han servido de nexo importante - a través del Consejo Mundial de Iglesias (WCC) - entre los movimientos de liberación nacional en Africa y el público alemán. Estas iglesias, si bien a veces han demostrado ser tibias y hesitantes, e incluso a causa de los problemas de violencia, altamente críticas de la política del WCC, han contribuido a destruir la creencia generalizada de que los regímenes pro bloque ocçidental siempre van en el interés de Alemania, sin que importe su estructura interna.

Para resumir este capítulo, las políticas hacia el Tercer Mundo están fuertemente determinadas por la peculiar historia de la República Federal de Alemania; la opinión pública está preparada para hacer ciertos sacrificios, pero la comunidad de desarrollo es escéptica frente a las medidas tradicionales; la formulación de políticas desde abajo sigue pautas bastante conservadoras, los grupos desarrollistas y los cientistas sociales poco ortodoxos están prácticamente excluidos de toda participación formal, y de alli que la respuesta de los principales políticos sea de vital importancia. Finalmente, en los últimos años los intereses alemanes han sido definidos en forma bastante estrecha, en términos de promoción de exportaciones, de asegurar el acceso a las materias primas y lograr un "buen clima" para las inversiones, dejando de lado intereses esclarecidos a largo plazo para promover un orden internacional basado en la autodependencia nacional, la justicia social, los derechos humanos y la erradicación de la pobreza en todo el mundo. El reciente empeoramiento en las relaciones Este-Oeste ha hecho retornar los intereses de bloque al primer plano de las políticas alemanas 
Norte-Sur. A fin de especificar más estos puntos generales, veremos en el próximo capítulo algunos de los temas principales en las relaciones Norte-Sur.

\section{TEMAS}

\section{1) La división internacional del trabajo}

Las relaciones de comercio internacional entre Alemania y el Tercer Mundo han estado dominadas tradicionalmente por un régimen liberal de importaciones para las materias primas, tarifas escalonadas que proporcionan una protección efectiva a las industrias procesadoras de productos, y promoción de exportaciones para maquinaria y otros bienes de inversión.

Como se expresó anteriormente, este modelo ha dejado su marca en la estructura internacional, y hace muy difícil el poder ajustarse a la prioridad surgida después de 1973 en las relaciones Norte-Sur; esto es, la energía o más precisamente el petróleo.

Desde la fundación de la República Federal de Alemania, las políticas sobre energía han significado una retirada ordenada desde el carbón local en favor de un petróleo barato, a fin de no obstruir la competividad internacional de la industria alemana.

Como no poseía una compañía petrolera nacional importante; el mercado alemán del petróleo estaba (y está) ampliamente abierto a las compañías petroleras internacionales, con solo un mínimum de superestructura internacional. Cuando los países de la OPEP plantearon los temas petroleros en los niveles gubernamentales, el gobierno alemán tuvo que crear primero los instrumentos políticos y administrativos para poder lidiar con ellos.

Sin embargo, la política interna general ha continuado dejando todo lo posible al mercado, sin interferir en decisiones de precios, excepto por razones antitrust. La política exterior con respecto al petróleo ha consistido en un bilateralismo cuidadoso, en procesos laboriosos de negociaciones multilaterales con la Comunidad Europea y la Agencia Internacional de Energía, y en varios intentos para introducir en diversos foros la energía en el diálogo Norte-Sur.

No obstante, las relaciones internacionales de energía no son contempladas como el modelo para un nuevo orden económico internacional, sino más bien como una excepción a la regla del libre comercio $^{12}$.

${ }^{12}$ Hermes. 
No es sorprendente que un país con la historia, la estructura política y los antecedentes económicos de la República Federal de Alemania sea un fiel abogado del libre comercio. Durante los treinta años de su existencia como estado, este país ha evolucionado desde un exportador marginal hasta el mayor proveedor mundial de exportaciones manufacturadas, manteniendo continuos excedentes comerciales y especializándose en algunas de las subramas más dinámicas e intensivas de tecnología, tales como maquinaria, equipos de transporte y productos químicos y farmacéuticos. La proporción de las exportaciones en el producto nacional bruto ha aumentado en forma sostenida, alcanzando el 20-25 por ciento en los últimos años.

La mayoría de las exportaciones van a otros países industrializados, lo que ha dado origen a la fórmula de que Alemania Occidental está interesada en que los países en desarrollo también se conviertan en países industriales. Sin embargo, puede haber una diferencia decisiva entre ser y llegar a ser un país industrializado. Los alemanes y sus vecinos europeos son los primeros en recordar las enormes inquietudes sociales, las tensiones internacionales y las guerras que acompañaron la industrialización tardía.

A pesar de la mezcla de sentimientos con respecto al lado político del desarrollo como un proceso, puede decirse que Alemania Occidental tiene un interés económico fuerte y permanente en el acceso a los mercados externos $y$, por poseer un sector de bienes de capital particularmente competitivo, en la mayor actividad de inversión mundial posible.

Sin embargo, en lo referente a la estructura económica interna de Alemania Occidental, la gran orientación hacia las exportaciones indụstriales ya ha producido el problema de la sobreindustrialización ${ }^{13}$. EI término se refiere a una comparación entre la República Federal de Alemania y otros países industriales con un ingreso por capita similar, pero con una menor participación de la industria en el PNB. Al mismo tiempo, se refiere a las tendencias futuras en cuanto a necesidades y demandas, progreso técnico, costos relativos del trabajo, tasas de intercambio y precios de las materias primas, en especial el petróleo y factores ecológicos, todos los cuales apuntan hacia una reducción relativa de la producción y el empleo en el sector industrial.

A pesar de que los cálculos para los años entre 1970 y 1976 indican que los desplazamientos por aumento de productividad sobrepasan los desplazamientos por importaciones de los países en desarrollo en un

${ }^{13}$ Schatz, Rogge. 
porcentaje que excede 20 a $1^{14}$, la "sobreindustrialización" es una característica importante al analizar las diversas opciones en la definición de los intereses alemanes frente al desarrollo del Tercer Mundo. Ella puede hacer necesarias estrategias muy diferentes: un proteccionismo defensivo, una política defensiva de avanzada o una retirada ordenada. De hecho, se usará probablemente una mezcla de las tres estrategias, de acuerdo a las ramas y a las firmas individuales. También tiende a disolver la gran coalición a la que nos hemos referido entre capital, trabajo, profesionales y ejecutivos, emanada de los años en que Alemania Occidental constituía la localización favorita para las inversiones industriales nacionales e internacionales. En un mundo en que el capital se ha vuelto cada vez más móvil y, aprovechando las ventajas derivadas de la competencia entre los países en lo referente a los incentivos impositivos, las exenciones frente a medidas de control ambiental, las fuentes de energía y la mano de obra baratas, los ingenieros y otros profesionales aspiran a transformar a Alemania Occidental de uno de los "talleres mundiales" en un taller de diseño internacional. Finalmente, el trabajador manual, que fue una vez el héroe de la reconstrucción alemana, el experto tras el crecimiento orientado hacia las exportaciones y el adulado consumidor de bienes manufacturados, está ahora en peligro de ser mirado en menos, como alguien cuyo trabajo puede o no puede ser protegido misericordiosamente, pero que ya no contribuye en forma significativa a la riqueza y al ingreso nacionales.

Como una reacción a estos desafíos que plantean las cambiantes condiciones económicas internacionales, han surgido nuevas ccircepciones con respecto a los beneficios de una división internacional del trabajo cada vez más profunda: en el nivel de la Comunidad Europea, como se destacó anteriormente, se hacen esfuerzos para unir nuevamente las estructuras políticas y económicas que se han ido apartando una de otras hasta el grado que la incongruencia empieza a crear un sentido de falta de poder y de impotencia. En el nivel nacional, las políticas en el campo de la ciencia y tecnología y de las actividades de investigación y desarrollo se están apartando gradualmente del "progreso", desde un no cuestionado énfasis en la energía nuclear, a expensas del carbón y de las fuentes alternativas de energía y desde una filosofía del desarrollo conducido por la tecnología ${ }^{15}$. Esta filosofía ya ha sido denunciada por la ex ministro de Cooperación Económica, María Schlei, como un "slogan traicionero", revelando que aquellos

${ }^{14}$ Livido, 51, y Dickie et al.

${ }^{15}$ Ver Hauff y Scharpf, y Bundesforschungsbericht (1979). 
que la usan no piensan en reducir la brecha entre el Norte y el Sur, sino más bien en perpetuarla, y que están preparados para dejar atrás a aquellos que no son capaces de trabajar en un "taller de diseño".

La gran atracción pública por iniciativas contrarias a las plantas nucleares como también la emergencia de partidos ecológicos, indican una creciente preocupación por aquellos que proponen la crítica de la tecnología o el movimiento de un "estilo de vida alternativo". Los nuevos valores - contenidos en sermones dominicales, declaraciones e informes ${ }^{16}$, pero denunciados diariamente por hechos y asercionesconducen a una creciente polarización entre el "stablishment" que cree en la división internacional del trabajo, en los grandes negocios y trabajos de las transnacionales, en el gran gobierno y la gran ciencia, y aquella parte de la nueva generación que adopta los nuevos valores. Sin embargo, en contraste con la generación estudiantil de 1967/68, esa gente joven no se ve envuelta en una lucha gigantesca, sino que es llamada con razón Aussteiger Jugend, juventud rebelde ${ }^{17}$.

A primera vista, este párrafo parece fuera de lugar en un capítulo sobre comercio internacional. Nó obstante, la definición de los intereses alemanes en el desarrollo del Tercer Mundo difiere tan agudamente entre los grupos en cuestión, de acuerdo a su visión del mundo (Weltanschaugen), en la cual el comercio internacional juega un papel radicalmente diferente, que se hace necesaria una referencia a esta discusión tan fundamental ${ }^{18}$.

Por lo general, se acepta que sería ingenuo ignorar las estructuras económicas globales, tecnológicas y de comunicación, y al mismo tiempo, que sería inhumano despreciar el pequeño mundo del individuo y su vida "alternativa" en canales informales. Si la consigna llega a ser "vivir con ambos", aceptando la combinación de tecnologías ${ }^{19}$, se arriba a un punto en que ni la división internacional del trabajo ni la noción de que lo pequeño es hermoso son puestos en un pedestal para ser venerados, sino que la selectividad junto con las necesidades e intereses se convierten en los principios orientadores.

Esta exigencia "bastante escéptica" de que se haga una cuidadosa evaluación, caso por caso, de las políticas de exportación y de importación puede ser contrastada con la adopción de un esquema para la

${ }^{16}$ Tales como la Declaración de Cocoyoc (1974), el informe Dag Hammarskjöld (1975), el Modelo de Bariloche (1976) y Laszlo et al. (1976).

${ }^{17}$ Richter.

${ }^{18}$ Weizsäcker (1978).

${ }^{19}$ Este concepto fue introducido por J. Galtung en UNCTAD (1978); ver también Galtung, Senghaas y Borges / Henke / Mutter / Nitsch en la Universidad Técnica de Berlín; y Nitsch (1978 a). 
creación de una economía social de mercado de alcance global, que fue presentada por el Consejo Académico Asesor a los Ministerios Federales de Economía en $1976^{20}$, y que contiene los trazos de la corriente del pensamiento económico profesional.

"Para poder aspirar a una asignación internacional dinámicamente eficiente de los escasos recursos disponibles", el autor aboga por una integración total de los países en desarrollo dentro de la división internacional del trabajo, por la remoción de las barreras comerciales tarifarias y no tarifarias que afectan a los productos de los países en desarrollo en los mercados de los países industriales, y por el establecimiento de "zonas de inversión garantida" en la forma de clubes abiertos de países preparados para proteger la inversión internacional privada del riesgo político. Separados del objetivo de la asignación, las metas de distribución deben ser mejoradas por medio de subvenciones masivas del orden de un $0,7 \%$ del PNB como un "primer paso" y planear un aumento en este porcentaje en una fecha posterior".

La crítica a esta propuesta se ha centrado en dos puntos ${ }^{21}$ : una economía global de mercado tiende a servir como un mecanismo de subasta, privando a los pobres hasta de los últimos bienes y asignando los recursos de acuerdo con la desigual distribución del poder global de compra. Segundo, es imposible en un mundo de naciones-estados lograr una redistribución efectiva mediante transferencias de ingresos en una escala tan masiva como para hacer comparable la economía internacional a la estructura de un estado del bienestar en lo interno. La asignación, de acuerdo con el poder comprador de los ricos, y la distribución de acuerdo con la generosidad de los mismos, vía transferencias unilaterales, serían estimadas como una doble imposición y no como una estrategia de desarrollo autodependiente realizada por el pueblo y para el pueblo en el Sur y en el Norte.

Entretanto, esta proposición ha sido postergada, pero todavía puede considerarse como típica de la clase de actitud globalista prevaleciente en el Consejo Asesor y en otras partes en Bonn, en tanto que en Bruselas hay que enfrentar los llamados a un "nuevo proteccionismo" 22 . En lo que respecta a los intereses globalistas del Tercer Mundo, la posición alemana en Bruselas puede ciertamente considerarse como una ayuda. Sin embargo, la retórica de Bonn sobre el libre comercio probablemente puede florecer tan solo porque los

\footnotetext{
${ }^{20}$ Ministerio Federal de Economía (citas de la traducción inglesa oficial).

${ }^{21}$ Ver Gagern.

22 Jeanneney.
} 
intereses proteccionistas y de control estatal alemanes son manejados discretamente en Bruselas.

\section{2) Inversión directa y corporaciones transnacionales}

La historia económica de la República Federal de Alemania y el fuerte convencimiento de su pertenencia al bloque occidental tiende a afirmar una creencia general en la mutualidad de intereses entre las corporaciones transnacionales, los países industriales y los países en desarrollo.

A mediados de los años 70, Alemania comenzó a ser un exportador neto de inversiones de capital, en lo concerniente a los flujos ${ }^{23}$. Con respecto a los inventarios de inversión directa, a fines de 1976 la inversión extr-ajera en la República Federal de Alemania (63.0 billones $\mathrm{DM}$ ) era todavía alrededor de un $50 \%$ más alta que la inversión alemana en el exterior ( 43.4 billones $\mathrm{DM})^{2 \cdot 1}$.

La rápida internacionalización de la economía alemana después de la Segunda Guerra Mundial, que al principio fue apoyada por la ayuda (Plan Marshall), iba mano a mano con un aumento espectacular y continuo del ingreso y con el pleno empleo, de tal manera que los flujos y reflujos de inversión externa eran fácilmente defendibles como una característica positiva de las economías modernas. El sistema alemán de coparticipación (Mitbestimmung) de los trabajadores y de sus sindicatos en el manejo de las firmas nacionales y extranjeras ha contribuido a la aceptación general de un sistema de estado benefactor neocapitalista, con las grandes corporaciones transnacionales. Sólo muy recientemente ha comenzado una discusión sobre la "exportación de trabajos". vía las exportaciones de capital; y al tener en sus directorios a miembros de los sindicatos, las firmas alemanas pueden resultar no ser tan móviles como sus competidoras puramente capitalistas. Pero todavía ésta es una pregunta sin respuesta.

La tendencia ya mencionada a transferir los principios del orden interno a la esfera internacional hace que parezca muy conservadora la posición de los intereses alemanes en el campo de las inversiones internacionales y de las corporaciones internacionales: el diálogo Norte-Sur no debe ser "una calle de una sola vía"; la protección de la propiedad privada como un principio de derecho internacional debería estar garantida. Es esencial para el desarrollo que haya un buen clima para la inversión extranjera; los códigos internacionales de con-

\footnotetext{
${ }^{23}$ Banco Central (1978).

${ }^{24}$ Banco Central (1979).
} 
ducta no deberían ser legalmente obligatorios, etc. En tanto que la frase "nuevo orden económico internacional" se entendió como si sólo implicara un mayor control nacional, el término fue considerado anatema por los intereses privados y por el gobierno. Sin embargo, han surgido nuevas interpretaciones, puesto que se ha comprendido que muchas de las características del NOEI pueden servir más para modernizar que para entorpecer la división internacional del trabajo.

El control sobre las corporaciones transnacionales recibió más atención y urgencia en la "segunda crisis del petróleo", después de los eventos en Irán. Se puede esperar que en el futuro se perciba en este campo una mayor mutualidad de intereses entre el Norte y el Sur, especialmente en el nivel de la comunidad europea ${ }^{25}$

Si la modernización a escala global y el control nacional o supranacional no son vistos como términos contradictorios sino como elementos del complejo problema de cómo obtener lo mejor de una economía globalista y del desarrollo tecnológico en un mundo político y jurídico de naciones-estados, se hace posible un enfoque más pragmático, a fin de alcanzar aquí también una combinación viable entre todos los elementos en juego.

Una sobria evaluación de los intereses debería comenzar de la tendencia capitalista de las firmas industriales hacia una globalización progresiva de la producción, el mercado y las finanzas. Las firmas alemanas, tomadas como un todo, poseen una característica especial, en la cual hay relativamente poca inversión directa en materias primas y más en manufacturas modernas; tales como automóviles, productos químicos y maquinarias. La inversión alemana directa está por consiguiente menos asociada con la "antigua" división internacional complementaria del trabajo, pero es bastante típica de la nueva "división" internacional de éste. Dentro de las economías internas de los países del Tercer Mundo, las firmás alemanas se concentran en bienes de consumo durạbles y maquinarias, y están en la avanzada con respecto a los mercados internacionales, promoviendo la exportación de manufacturas modernas de los países en desarrollo.

Esta pauta se ha visto en forma más clara en el caso del Brasil, donde se encuentra alrededor de un $40 \%$ de la inversión alemana directa en los países en desarrollo ${ }^{26}$. No es extraño que el "modelo brasileño" sea tan estimado en los círculos empresariales alemanes, como también en aquellos círculos académicos de Alemania que favorecen la integración

${ }^{25}$ Ver Holtz.

${ }^{26}$ Doellinger. 
mundial de los mercados como una estrategia de desarrollo ${ }^{27}$. Por otra parte, Brasil es el ejemplo favorito para los disasociacionistas y los estructuralistas, que llaman la atención sobre los problemas de la mala distribución del ingreso y la riqueza, y la persistencia de la pobreza y de la dependencia a pesar de las altas tasas de crecimiento del $\mathrm{PNB}^{28}$, - el típico ejemplo de una combinación desequilibrada y de una consecusión asimétrica de intereses.

Tal vez las características estructurales de la inversión externa alemana como promotora de la "nueva división internacional del trabajo" sean las responsables del hecho de que los líderes sindicales alemanes participen activamente en los movimientos sindicales internacionales (en lo que respecta a los sindicatos no comunistas), y que comprendan cada vez más que los intereses de los trabajadores alemanes radican en la elevación de los salarios y en una ampliación de los mercados nacionales de los países en desarrollo, antes que en una división entre el capital y el trabajo de las "ganancias neocoloniales excesivas" provenientes del exterior ${ }^{29}$.

Para resumir, en el campo de la inversión directa y de las corporaciones transnacionales hay una mutualidad de intereses limitada entre Alemania y el Tercer Mundo en lo que respecta al nivel globalista, el acceso a los mercados y a la tecnología moderna, como también a los intereses que presionen por una "nueva" división internacional del trabajo. El interés de control, que puede ser calificado de mutuo, se maneja en el nivel europeo. Los intereses para superar la pobreza y la marginalidad están todavía escondidos en declaraciones sobre el trabajo, y entran en conflicto con el carácter lujoso - para los patrones de los países en desarrollo- de la mayoría de los productos producidos por las firmas alemanas en el extranjero (mercancías duraderas y sus productos). Muchos observadores estarían de acuerdo en que "crecimiento sin desarrollo" pudiera ser el término adecuado para calificar aquello en lo cual están interesados los industriales alemanes en el exterior.

3) Ayuda

Desde el principio de la ayuda alemana para el desarrollo en los años cincuenta, el motivo humanitario y el difuso interés en el genuino desarrollo del Tercer Mundo han estado ligados a intereses más inme-

\footnotetext{
${ }^{27}$ Ver Parlamento Alemán (1974, 1977 y 1979), Donges y Müller-Ohlsen.

${ }^{28}$ Ver Senghaas (1977), Grabendorff y Nitsch (1977).

${ }^{29}$ Ver Buschmann, ICrFu, Elsehans.
} 
diatos, y compactos, tales como compartir el peso en el bloque occidental, el no reconocimiento de la República Democrática Alemana, la promoción de exportaciones e inversiones y la creciente respetabilidad internacional.

En 1971, el Gobierno Federal adoptó un "concepto de política para el desarrollo de la República Federal de Alemania" 30 , que enfatiza el "progreso económico y social en los países en desarrollo" y el "mejoramiento de las condiciones de vida de la población en esos países" como objetivos oficiales de la política alemana. El desarrollo del Tercer Mundo fue considerado necesario para "el propio interés de la República Federal de Alemania" 31 . Declaraciones de esa índole no eliminaron la creencia general en "el chorreo", pero acortaron la brecha entre el gobierno y la clientela altamente crítica de la política del desarrollo, que estaba influida principalmente por el movimiento estudiantil posterior a 1967 y por el pensamiento de la Nueva Izquierda.

Después de la renuncia de Erhard Eppler como Ministro de Cooperación Económica en 1974, la política gubernamental de desarrollo se alineó nuevamente con los intereses alemanes, definidos más estrechamente en el campo de la seguridad, en el abastecimiento de recursos críticos, en las exportaciones y en el empleo. Pero el pensamiento relacionado con el desarrollo trajo consigo el tema del desarrollo como interés alemán, relacionándolo con el movimiento ecológico y de "estilo de vida alternativo". Sin perder de vista los problemas específicos y su magnitud en el Tercer Mundo, "el desarrollo y la dirección del cambio tecnológico y social llegó a ser un cambio de perspectiva en los últimos años: en el capítulo comercial ya se ha mencionado la exigencia de una economía social de mercado a nivel internacional, con sistema de transferencias, comparable al sistema nacional de bienestar social" ${ }^{\text {"32. }}$. Por consiguiente a primera vista se puede esperar un amplio consenso con respecto a un aumento masivo de la ayuda.

Sin embargo, el desempeño de la ayuda ha sido decepcionante. El flujo neto de ayuda oficial para el desarrollo ha bajado del $0,45 \%$ del PNB en 1962 al 0,27 en 1977, lo que en parte puede ser atribuido al hecho de que la détente y la Ostpolitik han hecho a Alemania menos vulnerable a las presiones de los países en desarrollo. El porcentaje de la Asistencia Oficial para el Desarrollo (ODA) ha aumentado desde entonces a más del $0,3 \%$, debido a una nueva percepción frente a la nivelación de las capacidades ociosas en el Norte con las necesidades

\footnotetext{
${ }^{30}$ Ministerio de Cooperación Económica (1971).

${ }^{31}$ Eppler (1971).

${ }^{32}$ Ministerio Federal de Economia, Todenhöfer (1976).
} 
del Sur y debido, al mismo tiempo, a un clima más frío en las relaciones Este-Oeste. Pero, incluso si alguna vez se alcanzase la meta del $0,7 \% 0$ del $1 \%$, las magnitudes jamás se acercarán al $20-30 \%$ del PNB que se distribuye internamente a través del sistema oficial de bienestar.

A causa de los cientos de millones de gente pobre en el Tercer Mundo, la analogía - tan cara a los economistas alemanes, a los partidarios del libre comercio y a los administradores de la ayuda- entre el estado de bienestar interno y la economía global se vuelve terriblemente engañosa, cuando sugiere la viabilidad de una "economía social de mercado internacional" si pudiese tan solo aumentarse el presupuesto de la ayuda. El proceso presupuestario siempre está relacionado con la fuerza relativa de las clientelas, y esa es la razón por la cual no puede haber una redistribución masiva en favor de los no votantes; y, puede añadirse, ello es común aún menos probable en un regimen dictatorial. A pesar de no aspirar a proporciones magalómanas, la actitud positiva del pueblo alemán para ayudar a los pobres puede ser una combinación entre las ideas de transferencia de los desarrollistas y los economistas del mercado social y algún tipo de "impuesto al desarrollo". Una indicación adicional de la buena voluntad para aceptar un sistema internacional de transferencia puede ser visto en el hecho de que la analogía entre la asistencia para el desarrollo y el Plan Marshall ha llegado a ponerse de moda en Alemania Occidental (si bien particularmente con respecto al sur de Europa).

El problema consiste en cómo evitar la impresión, correcta o errónea, de que la ayuda es una transferencia de dinero de los pobres en los países ricos, a los ricos en los países pobres. Un primer paso es la declaración abierta de ayuda, entretando que una subvención destinada a los proyectos para combatir la pobreza minimiza sus lazos con otros intereses, generalmente más poderosos; un segundo paso es el retiro de la ayuda de la política exterior cotidiana, un tercero es la necesidad de competencia y publicidad para las agencias que canalizan la ayuda, a fin de evitar la burocratización y la corrupción ${ }^{33}$.

Aquí es suficiente destacar que los intereses generales alemanes para sobrepasar la pobreza y la marginalidad frente al respeto de los derechos humanos podrían ser servidos por alguna ayuda como la die la UNICEF de manera más directa, aunque sea muy modesta, y -en términos de la economía global-, un elemento muy marginal.

La magnitud de la ayuda puede aumentar considerablemente, y las asignaciones presupuestarias lo han hecho así en Alemania Occidental durante la reciente recesión, cuando estaban ligadas a otros intereses

${ }^{33}$ Para una propuesta detallada ver Hirschman y Bird (1968). 
tales como el empleo. La armonización de los intereses en mantener sus fuentes de abastecimiento y de empleo por parte de los países industriales con la demanda por maquinaria y otras importaciones por parte de los países en desarrollo han sido señalada como la panacea para superar las dificultades económicas actuales del mundo. En una audiencia parlamentaria, los institutos económicos alemanes de investigación han apoyado recientemente este punto de vista al unísono ${ }^{34}$, y el Consejo Alemán de Expertos en Economía ha adherido a é ${ }^{35}$. La duplicación de la transferencia neta de recursos de todos los países miembros del Comité de Asistencia para el Desarrolo (DAC) reducirá el número de desempleados en alrededor de un $17 \%$ y bajaría la tasa de desempleo en un $0.9 \%^{36}$.

En el caso de la ayuda concesional, las soluciones económicas son simples: gastos deficitarios y producción de bienes exportables para los países en desarrollo en vez de pirámides, armamentos o inversión privada interna. Como se destacó anteriormente, el problema radica en el proceso presupuestario democrático, que prohibe tales aumentos masivos, particularmente con una audiencia desarrollista que ha dejado de creer en que los bienes que podrían ser producidos y exportados por Alemania utilizando la actual capacidad industrial ociosa son necesariamente buenos para el desarrollo, y con un mercado internacional que detectaría correctamente en ello una atadura de los créditos y llamaría "dumping" a este tipo de promoción de exportaciones. Más aún, la transferencia de recursos en el Sur descansaría en grado superior en el ciclo de negocios del Norte.

Otra variante es la venta de bienes con créditos blandos, duros o cofinanciados. Aquí hay comprometidos intereses alemanes en el manejo del problema de la deuda, como también de los intereses comerciales. Por su propia naturaleza, el crédito sólo retrasa los problemas. De allí que el pago de los créditos esté asociado, ya bien con precios más elevados o con volúmenes mayores de importaciones, o bien con ambos en una fecha posterior. Si esto es imposible, o se ha hecho imposible, siempre se puede posponer un choque por medio de la prolongación, pero de una manera u otra habrá de venir una cancelación. El hecho de mantener en órbita deudas impagas hiere los intereses tanto de los deudores como de los acreedores, puesto que es inevitable la desconfianza mutua.

Siempre se pueden argumentar excepciones, pero en general la idea

\footnotetext{
${ }^{34}$ Parlamento Alemán (1979); Sachverständigenrat, 170-192.

${ }^{35}$ Instituto Alemán de Investigación Económica.

${ }^{36}$ UNIDO, 6.
} 
de unir capacidades ociosas aquí y necesidades obvias hace surgir muchas dudas.

Así sucede cón otros campos de interés y otros instrumentos; para una gama limitada $y$ hasta un cierto grado, casi todas las proposiciones tienen sentido, pero si se llevan muy lejos y están plagadas de demasiados objetivos y espectativas, tienen probabilidad de fallar.

\section{4) Otros temas}

Además del comercio, la inversión y la ayuda, deben añadirse otros temas a fin de llegar a obtener un perfil más o menos completo de los intereses alemanes y del desarrollo del Tercer Mundo.

En materias monetarias, la posición alemana (como la de la mayoría de los otros países industriales) es sólidamente conservadora, y contiene la marea para convertir al FMI en una institución para financiar el desarrollo, pero al mismo tiempo aumenta sus recursos y con ello su eficacia en tiempos de dificultades económicas para los países individuales.

De acuerdo con los manuales de economía, el sistema monetario internacional tiene como función emparejar el comercio y la inversión internacional; tal como en la política económica interna y las autoridades monetarias tienen que balancear la liquidez entre los dos peligros: el de la inflación, por una parte y el de la asfixia de la actividad económica por falta de liquidez, por la otra. No se puede esperar que las autoridades monetarias persigan objetivos tales como la autodependencia, los derechos humanos y la erradicación de la pobreza. No tienen ni el mandato, ni los instrumentos, ni el espíritu para ello, y ni siquiera el crecimiento económico ocupa un lugar importante en su lista de prioridades. Esa es la causa por la cual las políticas de los bancos centrales sólo pueden ser juzgadas en el contexto general de las políticas económicas internas.

Sin embargo, en el orden económico internacional existente el escenario institucional es todavía tan rudimentario que la autoridad monetaria - el FMI- puede usurpar un inmenso poder en la búsqueda de sus objetivos muy parciales y especializados. Por el hecho de ser la única agencia internacional con "dientes", el FMI puede reducir todas las instituciones de las Naciones Unidas, con la excepción del Consejo de Seguridad, a palabrería; y el Banco Mundial, que por estar orientado a largo plazo podría servir como un poder contrapuesto al FMI, (cuya orientación es a corto plazo) está siguiendo, en vez de controlando o balanceando, al FMI. 
Por consiguiente, la resultante sobre acentuación de los intereses globalistas alemanes a corto plazo y de los intereses occidentales de bloque a expensas de los intereses a largo plazo de Alemania Occidental en el mantenimiento de naciones - estados independientes y viables en el Tercer Mundo, en la erradicación de la pobreza y en los derechos humanos es, entre otras cosas, la consecuencia de la brecha institucional en la estructura internacional, y constituye un desafío para una estrategia visionaria de orden mundial.

Al seguir la premisa básica de este trabajo, vemos que no se intenta aquí denegar la legitimidad de intereses especiales tales como los que se manifiestan en el campo monetario, pero se confirma que los intereses especiales se enceguecen cuando son llevados demasiado lejos, y tienden a ahogar otros intereses igualmente legítimos. Los asuntos monetarios internacionales pueden constituir un caso apropiado.

Los intereses económicos alemanes en el comercio de armamentos son todavía bastante limitados, si se comparan con los de otros países industriales. Sin embargo, la importancia de estos intereses está creciendo por causa del problema del desempleo y porque la coproducción industrial con otros países está aumentando, de manera que el principio del gobierno federal de no permitir el envío de armas a "áreas de tensión" (Spanmengsgebiete) puede ser soslayado. Esta es la causa de que un nexo entre el desarme, las restricciones de transferencias de armas y el desarrollo podría calzar bastante bien dentro del perfil de los intereses alemanes. Pero puede estar terminándose el plazo para obtener triunfos en este campo.

El tema de las migraciones no es muy controvertido en la discusión pública en Alemania Occidental, pero ello no significa que no existen. problemas con raíces profundas. El argumento oficial es que la República Federal de Alemania no es un país de inmigración y que los trabajadores extranjeros están allí como invitados (Gastarbeiter). Sin embargo, esta interpretación oficial esconde más de lo que revela. De hecho, el status legal de los invitados y de sus familias, que no pertenecen a la Comunidad Económica Europea (CEE), es bastante inseguro: no son inmigrantes ni emigrantes contratados con un límite de tiempo definido. Durante la reciente recesión, los "trabajadores invitados" (incluyendo extranjeros de países miembros de la CEE), se convirtieron -en parte del "cojin" o "ejército de reserva" del empleo, y su participación en la fuerza laboral descendió considerablemente entre 1973 (cuando en el mes de octubre fueron detenidos nuevos contingentes provenientes de países que no pertenecían a la CEE) y 1977 , de 2.4 millones ( $10.7 \%$ de la fuerza laboral a 1.9 millones, o sea, el $8.8 \%$ ). Junto con una mejora de la situación del empleo cíclico a mediados de 
1979 , se oyeron nuevamente demandas provenientes de la industria de la construcción y de los negocios de hoteles y restaurantes, a fin de que ingresasen nuevos trabajadores de fuera de la CEE.

Individualizar los intereses en este campo no es tarea fácil. El interés de las empresas en un trabajo no especializado relativamente barato es bastante claro, pero la posición de los sindicatos, que favorecieron el ingreso de trabajadores invitados hasta 1973, necesita una explicación.

Los emigrantes ayudaron a estabilizar la tradicional estructura salarial, en la cual el trabajo organizado tiene un interés permanente. Con la expansión de las posibilidades educacionales, el trabajo no especializado se hizo escaso en Alemania Occidental y su precio en el mercado laboral se habia elevado en niveles cercanos a los de los trabajadores especializados. "Los alemanes ya no estaban preparados para esos trabajos sucios", se decía, y se olvidaba añadir "con un salario bajo". El cambio estructural en favor del trabajo no especializado ni organizado fue, por supuesto, percibido como un desafío a los trabajadores y a los empleados especializados y a sus sindicatos. Y puesto que éste es un problema estructural, el tema está destinado a revivir cada vez que se trate del pleno empleo.

Los frentes están claros, pero traspasan los límites tradicionales: aquellos que favorecen una estructura social jerárquica tenderán a favorecer la inmigración, en tanto que los que están a favor de una sociedad igualitaria y aquellos que compiten con los inmigrantres en los mercados laborales y habitacionales se opondrán a ella. Hasta ahora, las normas y valores constituyentes de la sociedad alemana se oponen a la heterogeneidad étnica. El principal objetivo político en los últimos siglos de la historia alemana ha sido la unidad alemana (Einheit), hasta llegar a las cimas racistas y a los horrores del Tercer Reich. La identidad alemana nunca ha sido comparable con la que los Ee.UU. tienen de ellos mismos, con sus valores simbolizados en la estatua de la libertad, y con su dificultad permanente para manejar los problemas raciales. Por todas estas razones, la inmigración del Tercer Mundo continuará siendo vista en el futuro como una excepción antes que como una regla, como puede verse con respecto a la titubeante recepción de refugiados y a la igualmente delicada cuestión del asilo político, un derecho humano que tiene su fundamento en el artículo 16 de la constitución de Alemania Occidental.

De allí que la migración no sea sólo una cuestión de contratar o no trabajadores en el extranjero, sino que se la ve cada vez más como la manifestación de una brecha creciente entre el Norte y el Sur en lo que respecta a los atractivos económicos y a los derechos humanos. Bajo este ángulo, un importante interés mutuo entre Alemania Occidental y 
el Tercer Mundo consiste en un rápido desarrollo interno y en el respeto a los derechos humanos en el Sur, de manera que países como Alemania Occidental no se vean invadidos por refugiados ni obligados a "fortificar" sus fronteras.

La lista de los intereses alemanes vis a vis al desarrollo del Tercer Mundo puede extenderse más, por ejemplo, al turismo, a la transferencia de tecnología, a la fuga de cerebros y al terrorismo, como también a problemas más globales tales como el desarme, la no proliferación, el equilibrio ecológico global y una estructura institucional conducente a la paz y el desarrollo en un orden mundial basado en el consenso en vez de en la fuerza.

\section{ADVERTENGIAS FINALES}

El perfil de los intereses alemanes en el desarrollo del Tercer Mundo puede ser agrupado en cuatro campos: los intereses globales, los intereses de bloque occidental, los intereses de control en los niveles nacionales y europeos y los intereses humanitarios en los derechos humanos y en la superación de la pobreza y la marginalidad.

El perfil resultante refleja el papel de la República Federal de Alemania como el principal exportador mundial de bienes de capital. Los intereses económicos globalistas son los más poderosos, y se articulan en la doctrina del libre comercio y de la creación de un clima favorable a la inversión, que a su vez tiende a borrar la línea entre las relaciones económicas globales, sin considerar los sistemas económicos y sociales, 'y las relaciones económicas dentro del bloque occidental. El interés en un orden mundial universal que comprenda todas las naciones no puede, por consiguiente, derivarse directamente de estrechos intereses económicos, sino que debe estar relacionado con la paz y el desarrollo como los objetivos principales, si bien abstractos, de políticas que carecen del apoyo de grupos compactos de. presión.

Cuando el perfil de los intereses alemanes se pone a la par con los requerimientos del desarrollo y de sus varias facetas e implicancias dinámicas, solamente se puede detectar una mutualidad inequívoca, si bien puede delinearse un gran campo de intereses comunes que están aguardando ser activados. Continúa siendo esencial la autodependencia en la articulación y en la búsqueda de intereses por ẹl lado del Tercer Mundo, puesto que es obvio que no se puede confiar en que los intereses alemanes sean lo suficientemente fuertes como para asegurar el desarrollo fuera de las fronteras de la República Federal de Alemania y de su unidad política y económica de mayor tamaño, la Comunidad Europea. 


\section{BIBLIOGRAFIA}

M. G. Adler-Karlsson, "Umgekehrter Utilitarismus oder neue Lebensformen in den entwickelten Ländern", Die Neue Internationale Wirtschaftsordnung in der Diskussion, UN-Texte 21, Bonn 1976, 93-102.

Agarwal, J.P., "Zur Struktur der Westdeutschen Direktinvestitionen in Entwicklungsländern", Die Weltwirtschaft, 1978, 1, 114-132.

S. Baron, H.H. Glismann y B. Stecher, Ökonomische Implikationen einer Neuen Weltwirtschaftsordnung, Forschungsauftrag des Bundesministers für.Wirtschaft, Kiel, Mar. 1977 (mimeo).

Bergedorfer Gesprächskreis, ed., Europa und die Weltwirtschaft-Politische und ökonomische Ansätze zur Lösung des Nord-Süd-Konfliktes, Hamburg 1977.

H. Besters, ed., Eine neue Ordnung der Weltwirtschaft?, Baden-Baden 1975.

J. Beyfuss, Aussenhandel mit Entwicklungsländern, Köln 1978.

Willy Brandt, "North-South Dialogue - A New Dimension of Peace Policy, Vierteljahresberichte, Sept. 1978, 73, 197-202.

L. J. Brinkhorst, "Die EWG und die Neue Weltwirtschaftsordnung", Die Neue Internationale Wirtschaftsordnung in der Diskussion, ONU-Texto 21, Bonn 1976, 73-84.

Ministerio de Investigación y Tecnología, ed., Bundesbericht Forschung VI, Bonn 1979.

Ministerio federal de Economía, ed., Fragen einer Neuen Weltwirtschaftsordnung, Studien-Reihe 15, Bonn 1976.

Ministerio de Cooperación Económica, ed., Die entwicklungspolitische Konzeption der Bundesrepublik Deutschland und die Internationale Strategie für die Zweite Entwicklungsdekade, Bonn 1971, y las ediciones revisadas de 1973 y 1975.

-, "Entwicklungspolitik: Parteien und gesellschaftliche Gruppen", Entwicklungspolitik, Material Nr. 61, Dec. 1978.

-, "Entwicklungszusammenarbeit zwischen der Bundesregierung und den christlichen Kirchen", Entwicklungspolitik, Material Nr. 62, Jan. 1979.

- Journalisten-Handbuch Entwicklungspolitik 1979, Bonn 1979. politik", Mitteilung für die Presse, 83/79, Dec. 20, 1979.

K. Buschmann, "Gefährdung oder Sicherung der Arbeitsplätze in den Industrieländern durch Industrialisierung in den Entwicklungsländern". Dokumente der Fachtagung "Entwicklungspolitik der SPD" am 1. und 2. Sept. 1977, Bonn 1977, 151-159.

Unión Demócrata Cristiana, ed., Bundesfachausschuß Entwicklungspolitik der CDU legt Thesen zur Nord-Süd-Politik vor, Pressemitteilung, Jan. 3, 1980.

Kenneth Dadzie, "Gemeinsame Interessen der Gewerkschaften in Industrie und Entwicklungsländern", Neue Entwicklungspolitik, 1978, 4, 5-8.

C. Deubner, U. Rehfeldt, F. Schlupp y G. Ziebura, Die Internationalisierning des Kapitals, Frankfurt/New York 1979.

Banco Central de Alemania, "Umschwung in der Bilanz der Direktinvestitionen", Monatsberichte der Deutschen Bundesbank, Oct. 1978, 30, 31-36. 
, "Stand der Direktinvestionen Ende 1976. Erste Ergebnisse einer neuen Statistik über die deutschen Directinvestitionen im Ausland und die ausländischen Direktinvestitionen in der Bundesrepublik Deutschland", Monatsberichte der Deutschen Bundesbank, Apr. 1979, 31, 26-40.

Deutsche Bundesregierung, "Politik der Zusammenarbeit mit den Entwicklungsländern - 17 Thesen der Bundesregierung", Entwicklungspolitik Spiegel der Presse, 1979, 23, 706-708.

Deutsche Gesellschaft für Technische Zusammenarbeit, ed., Science and Technology for Development. Contributions by the Federal Republic of Germany, Eschborn 1979.

Parlamento alemán, "Tätigkeit und entwicklungspolitischer Einflu $\beta$ deutscher multinationaler Unternehmen in Entwicklungsländern", Öffentliche Informationssitzung des Ausschusses für wirtschaftliche Zusammenarbeit, Nov. 11-12, 1974, Bonn 1974 (Bundestags-Drucksache).

—_, "Die Rohstoffproblematik im Nord-Süd-Verhältnis untęr besonderer Berücksichtigung des Integrierten Rohstoff-programms, Öffentliche Anhörung des Ausschusses für wirtschaftlicke Zusammenarbeit, May 23-25, 1977, Bonn 1977 (Bundestags-Drucksache).

—, "Nord-Süd-Verflechtung: Auswirkungen verstärkter Förderung der Länder der Dritten Welt durch öffentlichen und privaten Kapitaltransfer auf die Entwicklungsländer und die Industrieländer", Öffentliche Anhörung durch den Ausschu $\beta$ für wirtschaftliche Zusammenarbeit, Apr. 23-25, 1979, Bonn 1979 (Bundestags-Drucksache).

Deutscher Gewerkschaftsbund, XI. Ordenllicher Bundeskongress des DGB, Hamburg, 1978: Beschlüsse und Anträge, Düsseldorf 1978.

Deutsches Institut für Wirtschaftsforschung, "Aufstockung der Öffentlichen Entwicklungshilfe nützt Entwicklungs - und Industrieländern", DIW Wochenbericht, May 1979, 211-215.

Dag Hammarskjöld Report 1975, What now - Another Development, Stockholm 1975.

H. Dicke, H.H. Glismann, E.J. Horn and A.D.Neu, Beschäftigungswirkungen einer verstärkten Arbeitsteilung zwischen der Bundesrepublik und den Entwicklungsländern, Tübingen 1976.

C.v. Doellinger, "A Study in International Economic Relations: The BrazilianGerman Case", Ibero-Amerika-Insiitut für Wirtschafisforschung, Universität Göttingen, Diskussionsbeiträge $N^{\circ}$ 21, Jan. 1979.

J.B. Donges and L. Müller-Ohlsen, Außenwirtschaftsstrategien und Industrialisierung in Entwicklungsländern, Tübingen 1978.

J.B. Donges, G. Fels, A.D. Neu et al., Protektion und Branchenstruktur der westdeutschen Wirtschafl, Tübingen 1973.

P. Hrubesch y H. Lahmann, "Zur Einfuhrentwicklung der Bundesrepublik Deutschland von 1970 bis 1977", DIW-Wochenbericht, Aug. 1978.

Duve, Der Rassenkrieg findet nicht statt. Entwicklungspolitik zwischen Angst und Armut, Düsseldorf 1971.

F. Duve, ed., Technologie und Politik 11, Reinbek 1978. 
H. Elsenhans, "Für eine sozialreformeriche Ausgestaltung der NIWO", Entwicklung und Zusammenarbeit, 1979, 5, 19-25.

B. Engels, K.M. Khan y V. Matthies, Weltwirtschaftsordnung am Wendepunkt: Konflikt oder Kooperation? München 1975.

__ Wenig Zeit für die Dritte Welt, Stuttgart 1971.

, Ende oder.Wende, Stuttgart 1975.

E. Eppler, "Anfragen an unseren Lebensstil", epd Entwicklungspolitik, 1979, 7, $1-15$.

G. Fels, Der Standort Bundesrepublik im internationalen Wettbewerb, Kiel 1976.

I. Fetscher, Modelle der Friedenssicherung, München 1972.

D. Filip, R. Filip-Köhm, A. Geissler, C. Horn y H.S. Ziervogel, Abhängigkeit der Wirtschaft der Bundesrepublik Deutschland von Rohstoffimporten, Gutachten im Auftrag des Bundesministeriums für Wirtschaft (DIW and InfratestIndustria) Berlin/München 1976 (mimeo).

André Gunder Frank, Weltwirtschaft in der Krise, Reinbek 1978.

F. Fröbel, J. Heinrichs and O. Kreye, Die neue internationale Arbeitsteilung, Reinbek 1977.

M. Gagern, "Marktwirtschaft und Armenpflege im Weltmaßstab. Die Bundesrepublik Deutschland auf dem Holzweg" en J. Tinbergen, ed., Der Dialog Nord-Süd, Frankfurt 1977, 102-1 15.

E. Galeano, Las venas abiertas de América Latina, Montevideo 1971.

J. Galtung, Kapitalistische Großmacht Europa oder Die Gemeinschaft der Konzerne? "A Superpower in the Making", Reinbek 1973.

Genscher, "Perspektiven deutscher UN-Politik", Vereinte Nationen, 1979, 1, 1-6. H. Giersch, ed., The Changing International Division of Labour, Tübingen 1974. —, ed., Reshaping the World Economic Order, Tübingen 1977.

W. Grabendorff and M. Nitsch, Brasilien: Entwicklungsmodell und Außpolitik, München 1977.

M. and S. Greiffenhagen, Ein schwieriges Vaterland: Zur Politischen Kultur Deutschlands, München 1979.

W. Hager, "Angst vor der Dritten Welt?", Europa Archiv, 1975, 14, 471-476.

V. Hauff and F.W. Scharpt, Modemisierung der Volkswirtschafl, Frankfurt 1975.

H. Heck, "Technologische Flucht nach vorn. Export und Arbeitsplätze von morgen", Frankfurter Allgemeine Zeitung, 23.8.1975.

P. Hermes, "Außenpolitik und ökonomische Interessen", Außenpolitik, 1978, 3, 243-255.

A.O. Herrera and H.D. Scolnik, ed., Grenzen des Elends. Das Bariloche-Modell, Frankfurt 1977.

H. Hesse, "Die Diskussion über die Neuordnung der Weltwirtschaft", Wirtschaftsdienst, 1976, 10, 501-507.

U. Hiemenz y K.W. Schatz, "Internationale Arbeitsteilung als Alternative zur Ausländerbeschäftigung - Der Fall der Bundesrepublik Deutschland", Die Weltwirtschaft, 1977, 1, 35-58.

A.O. Hirschman y R.M. Bird, Foreign Aid: A Critique and a Proposal, Princeton 1968. 
Stanley Hoffmann, Primacy or World Order, New York 1978.

U. Holtz, Europa und die Multis, Baden-Baden 1978.

Ifo-Instituto de Investigación Económica, ed., "Neue Weltwirtschaftsordnung - Auswirkungen auf die deutsche Wirtschaft", ifo schnelldienst, July 15, 1977.

I. Illich, Selbsbegrezung, Reibek 1975.

Informationszentrum Dritte Welt Freiburg, ed., Entwicklungspolitik - Hilfe oder Ausbeutung? Die entwicklungspolitische Praxis der BRD und ihre wirtschaftlichen Hintergründe, Freiburg 1978.

International Confederation of Free Trade Unions, The ICFTU Development Charter, Brussels 1978.

J.M. Jeanneney, Pour un nouveau protectionnisme, Paris 1978.

Joint Conference of the Churches on Development in the Federal Republic of Germany, Justice and Solidarity in the International Economic Order, Hannover $y$ Bonn 1979.

R. Jonas y M. Tietzel, ed., Die Neuordnung der Weltwirtschaft, Bonn 1976.

D. Kebschull, W. Michalski y H.E. Scharrer, ed., Die neue Weltwirtschaftsordnung, Hamburg 1977.

M: Kreille, "West Germany: The Dynamics of Expansion", International Organization, 1977, 4, 775-808.

H. Kunst and H. Tenhumberg, ed., Soziale Gerechtigkeit und internationale Wirtschaftsordnung, München 1976.

Ervin Laszlo et al., Goals for Mankid, New York 1977.

K.W. Menck, ed., Wirtschaftliche Rezession und Entwicklungspolitik, Bonn 1976 (mimeo).

U. Müller-Plantenberg, "Die Bundesrepublik Deutschland und die Neue Weltwirtschaftsordnung", en V. Bennholdt-Thomsen et al., ed., Lateinamerika, Analysen und Berichte 2, Berlin 1978, 79-102.

G. Myrdal, The Challenge of World Poverty, New York 1970.

M. Nitsch, "Wissenschaft und Tecnologie für Entwicklung", LateinamerikaInstitut der Freien Universität Berlin, Diskussionspapiere $\mathrm{N}^{\circ}$ 8, Berlin 1978.

—_, "Zur Diskussion über die neue Weltwirtschaftsordnung", Stiftung Wissenschaft und Politik, ed., Polaritäl und Interdependez, Baden-Baden 1978, 317-335.

E. Noelle-Neumann, ed., Allensbacher Jahrbuch der Demoskopie 1976-77, München 1977.

Organization for Economic Cooperation and Development, Germany, Paris 1978.

H.E. Richter, "Was wir unseren Kindern vorleben. Die 'erwachsene' Gesellschaft und ihre Unfähigkeit, die Aussteigerjugend anzusprechen", Vorwärts, May 17, 1979, 16-17.

H. Riese, "Strukturwandel und unterbewertete Währung in der Bundesrepublik Deutschland", Konjunkturpolitik, 1978, 3, 143-169.

P.G. Rogge, "Weltwirtschaftliche Faktorallokation als Problem einzelstaatlicher Wirtschaftspolitik", Kyklos, 1979, 32, 331-349.

Sachverständigenrat zur Begutachtung der gesamtwirtschaftlichen Entwicklung, Jahresgutachten 1979/80, Bonn 1979. 
K.P. Sauvant y H. Hasenpflug, ed., The Netw International Economic Order, Frankfurt 1977.

K.F. Schade, "Ohne neues Währungssystem keine neue Wirtschaftsordnung", Die Neue Gesellschaft, Sept. 1978, 739-743.

K.W. Schatz, Wachstum und Strukturwandel der westdeutschen Wirlschaft im internationalen Verbund, Tübingen 1974.

M. Schlei, "Die Entwicklungspolitik der Bundesregierung, eingebunden in die Gesamtverantwortung der Europäischen Gemeischaft und der Industrieländer", Dokumente der Fachtagung "Entwicklungspolitik der SPD" am I. und 2. Sept: 1977, Bonn 1977, 144-150.

A. Schmidt, ed., Überwindung der Unterentwicklung, Frankfurt 1976.

Helmut Schmidt, "The Struggle for the World Product", Foreign Affairs, Apr. 1974, 438-451.

— Interview der Süddeutschen Zeîtung (Franz.Thoma), Süddeutsche Zeitung, 24.6.1975.

—_ "The International Economic Order and the North-South-Dialogue", Vierteljahresberichte, Dec. 1978, 74, 269-274.

K. Seitz, "Die Europäische Gemeinschaft in einer Welt des Übergans", EuropaArchiv, 1978, 16, 495-506.

-, "Die Verhandlungen über einen gemeinsamen Rohstoff-Fonds", Wirtschaftsdienst, 1978, 7, 93-97.

D. Senghaas y U. Menzel, ed., Multinationale Konzerne und Dritte Welt, Opladen 1976.

D. Senghaas, Weltwirtschaftsordnung und Entwicklungspolitik, Frankfurt 1977.

A. Skriver, Das Konzept der Hilfe ist falsch. Entwicklung in Abhängigkeit, Wuppertal 1977.

Technische Universität Berlin, ed., Technologische Grundlagenforschung für Entwicklungsländer, Berlin 1979.

L. Tindemans, "European Union - Informe por Mr. Leo Tindemans to the European Council", Bulletin of the European Communities, Supplemento I, 1976.

J.G. Todenhöfer, Wachstum für alle. Plädoyer für eine Internationale Soziale Marktwirtschaft, Stuttgart 1976.

—, Entrevista, Welt am Sonntag, Aug. 5, 1979.

W.G. Tyler, ed., Issues and Prospects for the New International Economic Order, Lexigton 1977.

UNCTAD, Development, Environment and Technology. Toward a Technology for SelfReliance (A Study by J. Galtung), TD/B/C.6/23, June 22, 1978.

UNIDO, Future Structural Changes in the Industry of the Federal Republic of Germany (Estudio preparado by K.W. Schatz y F. Wolter del Kiel Institute of World Economics en cooperación con UNIDO/ICIS. 103, March 20, 1979.

G.W. von Weizsäcker, Wege in der Gefahr, München 1976. Darmstadt 1978, 73-113.

F. Wolter, "Factor Proportions, Technology and West German Industries International Trade Patterns", Weltwirtschaftliches Archiv, 1977, 113, 250267. 